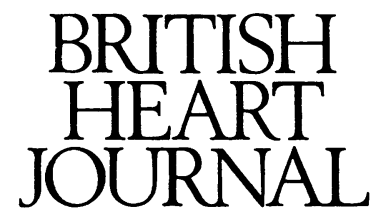

Editorial

\title{
Should coronary angiography be performed in district hospitals?
}

Arrangements for the optimal distribution and delivery of specialist cardiological care are haphazard to the point of being kaleidoscopic. Changes both in the type of cardiac disorders requiring specialist assessment and also in technological advances-most strikingly in echocardiography and exercise testing-have effectively devolved triage to district general hospitals. ${ }^{1}$ With increasing indications for intervention the question whether coronary angiography should be provided by distict hospitals will undoubtedly have to be answered in the next decade.

Questions about the provision of facilities are invariably complex because they encompass geographical, financial, clinical, professional, and political considerations. In such complex discussions, facts are often conspicuous by their absence. The article from St George's Hospital in the current issue (page 74), however, describes the current risks of coronary angiography and the extent to which immediate access to cardiac surgery can reduce mortality associated with the unavoidable complications of the investigation. $^{2}$

The results show that case selection rather than the seniority of the operator was a major determinant of complications. Also the brachial approach was associated with a higher incidence of dissection of the main stem of the left coronary artery. This may be because the brachial approach is usually chosen for patients with clinically overt peripheral vascular disease, who are more likely to have severe coronary disease. The pre-shaped coronary artery catheters that were used from the arm may also have contributed to this association with dissection. Since the $\mathrm{St}$ George's study 6 French gauge catheters have become more generally used and this refinement may have reduced the incidence of dissection of the main stem of the left coronary artery.

Dr Stewart and his colleagues have confirmed that potentially fatal problems can still arise after coronary angiography and that emergency surgery is important in ameliorating the long term effect of these risks. There can be little doubt that to be effective surgery must be performed as soon as possible, with 4 hours being the limit for successful revascularisation. ${ }^{3}$ Against this, we have to offset the likelihood of an increase in mortality if patients have to join a waiting list at major regional centres rather than being investigated more quickly in a district hospital. Would locally based diagnostic coronary angiography hasten life saving surgical intervention for left main stem or three vessel coronary artery disease in patients without either unstable symptoms or severely impaired left ventricular function? The St George's study quantifies one side of the equation-that is, the risks of investigation without surgical cover. Now the other side deserves attention.

There is a third question associated with the provision of coronary angiography in district hospitals. If patients are to face an inevitably increased risk, albeit small in absolute terms, will the quality of images from the procedure in the district hospital without surgical cover justify this risk by improving and expediting management decisions? We recently looked at image quality on standard video tape recordings with an image intensifier at Newham General Hospital. ${ }^{4}$ Vessel patency after thrombolysis was accurately assessed by the district hospital video images. The sensitivity for important disease in the circumflex artery was only $40 \%$ when the video images were compared with regional centre live film. This would inevitably have led to incorrect management decisions being made because of the inferior image quality. The cardiac surgeon who also assessed the video tapes reached a similar conclusion.

The group from St George's Hospital has shown that any trend towards devolution of diagnostic coronary angiography away from the protective environment of on-site cardiac surgical cover will add a risk to the procedure. Such a change in the provision of resources should be considered only if appropriate capital investment is made to ensure that the diagnostic value of the resultant angiogram is sufficiently high to enable sound clinical decisions to be made and if it can be shown that the improved access to angiography is likely to reduce waiting list mortality.

The London Hospital, PETER MILLS

Whitechapel,

London E1 $1 B B$

1 Ross J, Gilpin E, Madsen E, et al. A decision scheme for coronary angiography after acute myocardial infarction. Circulation 1989;79: 292-303.

2 Stewart JT, Gray HH, Ward DE, Pumphrey CW, Redwood DR, Parker DJ. Major complications of coronary arteriography: the place of cardiac surgery. Br Heart J 1990;63:74-7.

3 Spencer FC. A critique of emergency and urgent operations for complications of coronary artery disease. Circulation 1989;79(suppl I):160-2.

4 Ranjadayalan K, Mills P, Sprigings D, Mourard K, Magee P, Timmis A. Coronary arteriography in the radiology department of a district general hospital: feasibility, safety, and diagnostic accuracy. Br Med J 1990;300: (in the press). 